УДК 532.13

\title{
О единственности классического решения начально- краевой задачи течения вязкоупругой среды Максвелла с нулевыми скоростями на границе
}

\author{
А.Г. Петрова \\ Алтайский государственный университет (Барнаул, Россия)
}

\section{On the Uniqueness of the Classical Solution of the Initial- boundary Value Problem for a Viscoelastic Maxwell Medium Flow with Zero Velocities at the Boundary}

\author{
A.G. Petrova \\ Altai State University (Barnaul, Russia)
}

\begin{abstract}
Рассматривается система уравнений с вращательной производной Яуманна, описывающая движения вязкоупругой несжимаемой среды Максвелла в ограниченной области трехмерного пространства в приближении Стокса (без конвективных слагаемых как в уравнении движения, так и в реологическом соотношении). Выбор объективной производной Яуманна в реологическом соотношении обусловлен наличием энергетического тождества, справедливость которого не удается доказать для случаев использования верхней и нижней конвективных производных в качестве объективных производных. Доказывается единственность классического решения начально-краевой задачи с условием прилипания на границе области течения в классе достаточно гладких функций в предположении, что система обладает четырьмя различными вещественными звуковыми характеристиками. Доказательство основано на применении интегральных оценок и использовании условия гиперболичности системы. Для наглядности выкладки проводятся для двумерного случая, однако нигде не используются свойства двухмерности. Отличия от трехмерного случая носят чисто технический характер. Отмечается, что наличие конвективных членов в уравнениях движения не препятствует доказательству единственности. Единственность первоначально доказывается для малого промежутка времени, затем стандартным образом утверждение распространяется на промежуток произвольной длины.

Ключевые слова: несжимаемая вязкоупругая среда Максвелла, производная Яуманна, начально-краевая задача, единственность.
\end{abstract}

DOI 10.14258/izvasu(2018)4-17
A system of equations with a rotational Jaumann derivative describing the motion of a viscoelastic incompressible Maxwell medium in a bounded region of three-dimensional space in the Stokes approximation (without convective terms in both the equation of motion and the rheological relation) is under consideration. The choice of the objective derivative of Jaumann type in the rheological ratio is due to the presence of an energy identity, the validity of which cannot be proved for the cases of using the upper and lower convective derivatives as objective derivatives. The uniqueness of the classical solution of the initial-boundary value problem with the adherence condition on the boundary of the flow domain in the class of sufficiently smooth functions under the assumption that the system has four distinct real sound characteristics is proved. The proof is based on the application of integral estimates and the use of the hyperbolicity condition of the system. For the sake of clarity, the calculations are carried out for the two-dimensional case, however, nowhere does it use the properties of two-dimensionality. Differences from the three-dimensional case are purely technical in nature. It is noted that the presence of convective terms in the equations of motion does not prevent the proof of uniqueness. Uniqueness is initially proved for a small interval of time, then in the standard way, the statement is extended to an interval of arbitrary length.

Key words: viscoelastic incompressible Maxwell medium, Jaumann derivative, initial-boundary-value problem.

*Работа выполнена при финансовой поддержке Российского фонда фундаментальных исследований (код проекта 1601-00127). 
Постановка задачи. Движению вязкоупругих сред посвящено огромное количество работ (см. монографии [1-4] и библиографию к ним). Математические вопросы движения несжимаемой вязкоупругой среды Максвелла рассматривались, в частности, в статьях [5-10]. Однако в подавляющем большинстве публикаций рассматривается двумерный случай, а в качестве объективной производной выбирается более удобная верхняя конвективная производная. Данная работа посвящена простейшей начально-краевой задаче для движения в пространстве, причем в качестве объективной производной выбирается производная Яуманна. Материальными характеристиками вязкоупругой среды являются ее плотность $\rho$, динамическая вязкость $\mu$ и время релаксации $\tau$. Эти величины далее полагаются постоянными; $\mathbf{v}-$ вектор скорости; $\mathrm{p}-$ отклонение давления от некоторого среднего значения $\mathrm{p}_{0}>0$; D - тензор скоростей деформаций; $\mathrm{S}-$ вязкоупругая составляющая тензора напряжений; W антисимметричная часть тензора $\nabla \mathbf{v}$. Система урав- нений, описывающих движение несжимаемой вязкоупругой среды Максвелла с производной Яуманна в качестве инвариантной производной, имеет вид [1]:

$$
\begin{gathered}
\operatorname{div} \mathbf{v}=0, \\
\rho\left(\mathbf{v}_{t}+\mathbf{v} \cdot \nabla \mathbf{v}\right)=-\nabla p+\operatorname{div} \mathbf{S}, \\
\tau\left(\mathbf{S}_{t}+\mathbf{v} \cdot \nabla \mathbf{S}-\mathbf{W} \cdot \mathbf{S}+\mathbf{S} \cdot \mathbf{W}\right)+\mathbf{S}=2 \mu \mathbf{D}
\end{gathered}
$$

Отметим, что система совместна с дополнительным соотношением

$$
\operatorname{Tr} \mathbf{S}=0
$$

при условии, что это соотношение выполнено при $\mathrm{t}=0$ [7].

Выбор объективной производной Яуманна в реологическом соотношении обусловлен наличием энергетического тождества $[7,8,10]$

$$
\frac{d}{d t} \int_{\Omega} \frac{1}{2}\left(\rho|\mathbf{v}|^{2}+\frac{\tau}{2 \mu} \mathbf{S}: \mathbf{S}\right) d \Omega=\int_{\Sigma} \mathbf{v}(-p \mathbf{n}+\mathbf{S} \cdot \mathbf{n}) d \Sigma-\frac{1}{2 \mu} \int_{\Omega} \mathbf{S}: \mathbf{S} d \Omega,
$$

где $\mathbf{n}-$ внешняя нормаль к поверхности $\Sigma$, ограничивающей объем $\Omega$, справедливость которого удается доказать лишь в этом случае. Если

$$
\left.\mathbf{v}\right|_{\partial \Omega \times[0, t)}=0,
$$

энергетическое тождество (3) приобретает вид

$$
\frac{d}{d t} \int_{\Omega_{t}} \frac{1}{2}\left(\rho|\mathbf{v}|^{2}+\frac{\tau}{2 \mu} \mathbf{S}: \mathbf{S}\right) d \Omega=-\frac{1}{2 \mu} \int_{\Omega_{t}} \mathbf{S}: \mathbf{S} d \Omega .
$$

На основе этого тождества в работе [10] доказана единственность классического решения линеаризованной на состоянии покоя задачи движения вязкоупругой среды Максвелла с нулевыми скоростями на границе области. Отметим, что в этой же статье найдены характеристики системы (1) в трехмерном случае.

Будем предполагать выполненным условие «гиперболичности» системы [10]:

$$
m=\frac{\mu}{\tau} \geq 6 \max \left\{S_{i j}\right\},
$$

Отметим, что для классического решения это условие выполнено на малом интервале времени, если в начальный момент имело место строгое неравенство.

Будем исследовать классическое решение задачи с условиями прилипания на границе $\Sigma$ области течения $\Omega$ в случае отсутствия конвективных членов, когда система управляющих уравнений принимает вид

$$
\operatorname{div} \mathbf{v}=0, \rho \mathbf{v}_{t}=-\nabla p+\operatorname{div} \mathbf{S}, \tau\left(\mathbf{S}_{t}-\mathbf{W} \cdot \mathbf{S}+\mathrm{S} \cdot \mathbf{W}\right)+\mathrm{S}=2 \mu \mathbf{D}
$$

Теорема. Классическое решение задачи (6), (4), дополненной начальными данными для всех искомых функций, при условиях (2), (5) единственно в классе функций

$$
\begin{aligned}
& \mathbf{v} \in\left(C^{2}(\bar{\Omega} \times[0, T])\right)^{3}, \mathbf{S} \in\left(C^{1}(\bar{\Omega} \times[0, T])\right)^{6} . \\
& \mathbf{v}=(u, v), \mathbf{S}=\left(\begin{array}{cc}
a & b \\
b & -a
\end{array}\right), \quad \mathbf{v}=\mathbf{v}^{(1)}-\mathbf{v}^{(2)}, a=a^{(1)}-a^{(2)}, b=b^{(1)}-b^{(2)} . \\
& \text { Сиский характер. } \\
& \text { Система (6) в двумерном случае для разности двух } \\
& \text { решений примет вид: }
\end{aligned}
$$

Доказательство теоремы. В целях упрощения выкладок проведем доказательство для двумерного случая, нигде не пользуясь свойствами двумерности. Отличия от трехмерного случая носят чисто техни- 


$$
\begin{gathered}
\rho v_{t}+p_{y}=b_{x}-a_{y}, \\
a_{t}+a / \tau=2 m u_{x}+b\left(u_{y}^{(1)}-v_{x}^{(1)}\right)+b^{(2)}\left(u_{y}-v_{x}\right), \\
b_{t}+b / \tau=m\left(u_{y}+v_{x}\right)-a\left(u_{y}^{(1)}-v_{x}^{(1)}\right)-a^{(2)}\left(u_{y}-v_{x}\right) .
\end{gathered}
$$

Проделаем ту же процедуру, что и при получении интегрального тождества (3) $[6,7,9]$. Умножим (10) на $a,(11)$ на $b$, сложим и проинтегрируем по области течения; умножим (8) на $u$, (9) на $v$, проинтегрируем по области течения и сложим с использованием (7). Комбинация результатов этих действий приводит к соотношению:

$$
\frac{d}{d t} \int_{\Omega} \frac{1}{2}\left(m \rho|\mathbf{v}|^{2}+\frac{1}{2} \mathbf{S}: \mathbf{S}\right) d \Omega+\frac{1}{2 \tau} \int_{\Omega} \mathbf{S}: \mathbf{S} d \Omega=\int_{\Omega}\left(a b^{(2)}-b a^{(2)}\right)\left(u_{y}-v_{x}\right) d \Omega .
$$

Интегрируя по времени с учетом того, что начальные условия для всех функций однородны, приходим

к равенству:

$$
\int_{\Omega} \frac{1}{2}\left(m \rho|\mathbf{v}|^{2}+\frac{1}{2} \mathbf{S}: \mathbf{S}\right) d \Omega+\frac{1}{2 \tau} \int_{0}^{t} d t \int_{\Omega} \mathbf{S}: \mathbf{S} d \Omega=\int_{0}^{t} d t \int_{\Omega}\left(a b^{(2)}-b a^{(2)}\right)\left(u_{y}-v_{x}\right) d \Omega .
$$

Для оценки правой части нужна оценка квадрата градиента вектора скорости. Чтобы ее получить, продифференцируем уравнения (8) и (9) по времени и сложим результаты с этими же уравнениями, умноженными на коэффициент $\tau^{-1}$. Получим векторное уравнение

$$
\rho\left(\mathbf{v}_{t t}+\mathbf{v}_{t} / \tau\right)+\nabla\left(p_{t}+p / \tau\right)=\operatorname{div}\left(\mathbf{S}_{t}+\mathbf{S} / \tau\right) .
$$

Распишем это векторное уравнение для двумерного случая в виде системы двух скалярных:

$$
\begin{aligned}
& \rho\left(u_{t t}+u_{t} / \tau\right)+\left(p_{t}+p / \tau\right)_{x}=\left(m-a^{(2)}\right) \Delta \mathrm{u}-b^{(2)} \Delta \mathrm{v}+ \\
& +\left(b_{x}^{(2)}-a_{y}^{(2)}\right)\left(u_{y}-v_{x}\right)+\left(b\left(u_{y}^{(1)}-v_{x}^{(1)}\right)\right)_{x}-\left(a\left(u_{y}^{(1)}-v_{x}^{(1)}\right)\right)_{y} \\
& \rho\left(v_{t t}+v_{t} / \tau\right)+\left(p_{t}+p / \tau\right)_{y}=\left(m+a^{(2)}\right) \Delta v-b^{(2)} \Delta u+ \\
& +\left(a_{x}^{(2)}+b_{y}^{(2)}\right)\left(u_{y}-v_{x}\right)-\left(b\left(u_{y}^{(1)}-v_{x}^{(1)}\right)\right)_{y}-\left(a\left(u_{y}^{(1)}-v_{x}^{(1)}\right)\right)_{x}
\end{aligned}
$$

Далее нужно уравнение (14), умноженное на $u_{t}$, сложить с (15), умноженным на $v_{t}$, и результат проинтегрировать по цилиндру $\Omega \times(0, t)$. Используя фор-

мулу Остроградского - Гаусса и условие (4), приходим к равенству

$$
\begin{aligned}
& \int_{\Omega} \rho \frac{\left|\mathbf{v}_{t}\right|^{2}}{2} d \Omega+\int_{0}^{t} \int_{\Omega} \rho \frac{\left|\mathbf{v}_{t}\right|^{2}}{\tau} d \Omega d t+\int_{\Omega}\left(\left(m-a^{(2)}\right) \frac{|\nabla u|^{2}}{2}+\left(m+a^{(2)}\right) \frac{|\nabla v|^{2}}{2}-b_{2} \nabla u \cdot \nabla v\right) d \Omega- \\
& -\int_{\Omega}\left(b\left(u_{x}-v_{y}\right)-a\left(u_{y}+v_{x}\right)\right)\left(u_{y}^{(1)}-v_{x}^{(1)}\right) d \Omega= \\
& =-\int_{0}^{t} \int_{\Omega}\left(b_{t}\left(u_{x}-v_{y}\right)-a_{t}\left(u_{y}+v_{x}\right)\right)\left(u_{y}^{(1)}-v_{x}^{(1)}\right) d \Omega d t- \\
& -\int_{0}^{t} \int_{\Omega}\left(b\left(u_{x}-v_{y}\right)-a\left(u_{y}+v_{x}\right)\right)\left(u_{y t}^{(1)}-v_{x t}^{(1)}\right) d \Omega d t- \\
& -\int_{0}^{t} \int_{\Omega}\left(a_{t}^{(2)} \frac{|\nabla \mathbf{v}|^{2}}{2}-b_{t}^{(2)} \nabla u \nabla v+a_{x}^{(2)}\left(u_{x} u_{t}+u_{y} v_{t}-2 v_{x} v_{t}\right)+a_{y}^{(2)}\left(v_{x} u_{t}-v_{y} v_{t}\right)\right) d \Omega d t- \\
& -\iint_{x}\left(b_{x}^{(2)}\left(u_{x} v_{t}+u_{y} u_{t}\right)+b_{y}^{(2)}\left(2 v_{y} u_{t}+u_{y} v_{t}-v_{x} v_{t}\right)\right) d \Omega d t+ \\
& +\int_{0}^{t} \int_{\Omega}\left(b_{t}\left(u_{x}-v_{y}\right)-a_{t}\left(u_{y}+v_{x}\right)\right)\left(u_{y}^{(1)}-v_{x}^{(1)}\right) d \Omega d t .
\end{aligned}
$$


Заметим, что в силу уравнений (10), (11) производные по времени от компонент тензора $\mathbf{S}$ выражаются через сами компоненты и компоненты градиента скорости, причем их выражение не содержит производных от компонент тензора по пространственным переменным, вследствие чего получаем оценку:

$$
\begin{aligned}
& \int_{\Omega} \rho \frac{\left|\mathbf{v}_{t}\right|^{2}}{2} d \Omega+\int_{0}^{t} \int_{\Omega} \rho \frac{\left|\mathbf{v}_{t}\right|^{2}}{\tau} d \Omega d t+\int_{\Omega}\left(\left(m-a^{(2)}\right) \frac{|\nabla u|^{2}}{2}+\left(m+a^{(2)}\right) \frac{|\nabla v|^{2}}{2}-b_{2} \nabla u \cdot \nabla v\right) d \Omega- \\
& -\int_{\Omega}\left(b\left(u_{x}-v_{y}\right)-a\left(u_{y}+v_{x}\right)\right)\left(u_{y}^{(1)}-v_{x}^{(1)}\right) d \Omega \leq \int_{0}^{t} \int_{\Omega} L\left(\rho \frac{\left|\mathbf{v}_{t}\right|^{2}}{2}+\frac{|\nabla \mathbf{v}|^{2}}{2}+\mathbf{S}: \mathbf{S}+|\mathbf{v}|^{2}\right) d \Omega d t .
\end{aligned}
$$

Применим неравенство Коши с эпсилон следнем интеграле левой части неравенства (16): для оценки подынтегрального выражения в по-

$$
\left|b\left(u_{x}-v_{y}\right)-a\left(u_{y}+v_{x}\right)\right|\left|u_{y}^{(1)}-v_{x}^{(1)}\right| \leq k\left(\frac{b^{2}+a^{2}}{2 \varepsilon}\right)+k \varepsilon\left(|\nabla u|^{2}+|\nabla v|^{2}\right)
$$

где $k=\max \left|u_{y}^{(1)}-v_{x}^{(1)}\right|$. Выберем $\varepsilon$ так, чтобы выполнялись неравенства

$$
m-2 \varepsilon k-\left|a^{(2)}\right| \geq \varepsilon, \quad(m-2 \varepsilon k)^{2}-\left(a^{(2)}\right)^{2}-\left(b^{(2)}\right)^{2} \geq \varepsilon
$$

Это возможно вследствие условия (5). Оценивая неравенства (16) снизу, получим: с использованием (17) левую часть интегрального

$$
\begin{gathered}
\int_{\Omega} \rho \frac{\left|\mathbf{v}_{t}\right|^{2}}{2} d \Omega+\int_{0}^{t} \int_{\Omega} \rho \frac{\left|\mathbf{v}_{t}\right|^{2}}{\tau} d \Omega d t+\int_{\Omega}\left(\left(m-a^{(2)}-2 k \varepsilon\right) \frac{|\nabla u|^{2}}{2}+\left(m+a^{(2)}-2 k \varepsilon\right) \frac{|\nabla v|^{2}}{2}-b_{2} \nabla u \cdot \nabla v\right) d \Omega- \\
\leq \int_{\Omega} k\left(\frac{b^{2}+a^{2}}{2 \varepsilon}\right) d \Omega+\int_{0}^{t} \int_{\Omega} L\left(\rho \frac{\left|\mathbf{v}_{t}\right|^{2}}{2}+\frac{|\nabla \mathbf{v}|^{2}}{2}+\mathbf{S}: \mathbf{S}+|\mathbf{v}|^{2}\right) d \Omega d t .
\end{gathered}
$$

Здесь через L обозначен максимум норм всех Продолжая оценивать левую часть снизу (16) с исфункций в выбранных классах. пользованием (17), (18), приходим к оценке

$$
\begin{gathered}
\int_{\Omega} \rho \frac{\left|\mathbf{v}_{t}\right|^{2}}{2} d \Omega+\int_{0}^{t} \int_{\Omega} \rho \frac{\left|\mathbf{v}_{t}\right|^{2}}{\tau} d \Omega d t+\varepsilon \int_{\Omega}\left(\frac{|\nabla u|^{2}}{2}+\frac{|\nabla v|^{2}}{2}\right) d \Omega-\int_{\Omega} k\left(\frac{b^{2}+a^{2}}{2 \varepsilon}\right) d \Omega \leq \\
\leq \int_{0}^{t} \int_{\Omega} L\left(\rho \frac{\left|\mathbf{v}_{t}\right|^{2}}{2}+\frac{|\nabla \mathbf{v}|^{2}}{2}+\mathbf{S}: \mathbf{S}+|\mathbf{v}|^{2}\right) d \Omega d t
\end{gathered}
$$

Из последней оценки следует неравенство

$$
\begin{gathered}
\int_{\Omega} \rho \frac{\left|\mathbf{v}_{t}\right|^{2}}{2} d \Omega+\varepsilon \int_{\Omega}\left(\frac{|\nabla u|^{2}}{2}+\frac{|\nabla v|^{2}}{2}\right) d \Omega-\int_{\Omega} k\left(\frac{b^{2}+a^{2}}{2 \varepsilon}\right) d \Omega \leq \\
\leq t L \int_{\Omega} \rho \frac{\left|\mathbf{v}_{t}\right|^{2}}{2} d \Omega+t L \int_{\Omega}\left(\frac{|\nabla u|^{2}}{2}+\frac{|\nabla v|^{2}}{2}\right) d \Omega .
\end{gathered}
$$


Умножим (13) на $\mathrm{k} / \varepsilon$ и, складывая почленно с (19), получим:

$$
\begin{gathered}
\int_{\Omega} \rho \frac{\left|\mathbf{v}_{t}\right|^{2}}{2} d \Omega+\varepsilon \int_{\Omega}\left(\frac{|\nabla u|^{2}}{2}+\frac{|\nabla v|^{2}}{2}\right) d \Omega+\frac{k}{2 \varepsilon} \int_{\Omega}\left(m \rho|\mathbf{v}|^{2}+\mathbf{S}: \mathbf{S}\right) d \Omega+ \\
+\frac{k}{\varepsilon} \frac{1}{2 \tau} \int_{0}^{t} d t \int_{\Omega} \mathbf{S}: \mathbf{S} d \Omega \leq t L \int_{\Omega} \rho \frac{\left|\mathbf{v}_{t}\right|^{2}}{2} d \Omega+t L \int_{\Omega}\left(\frac{|\nabla u|^{2}}{2}+\frac{|\nabla v|^{2}}{2}\right) d \Omega+ \\
+\frac{k}{\varepsilon} t L \int_{\Omega} \frac{1}{2}\left(m \rho|\mathbf{v}|^{2}+\mathbf{S}: \mathbf{S}\right) d \Omega .
\end{gathered}
$$

Последняя оценка приводит к неравенству

$$
(1-t L)\left(\int_{\Omega} \rho \frac{\left|\mathbf{v}_{t}\right|^{2}}{2} d \Omega+\varepsilon \int_{\Omega}\left(\frac{|\nabla u|^{2}}{2}+\frac{|\nabla v|^{2}}{2}\right) d \Omega+\frac{k}{\varepsilon} \int_{\Omega} \frac{1}{2}\left(m \rho|\mathbf{v}|^{2}+\mathbf{S}: \mathbf{S}\right) d \Omega\right) \leq 0
$$

из которой при $t<1 / L$ следует, что

$$
\int_{\Omega} \rho \frac{\left|\mathbf{v}_{t}\right|^{2}}{2} d \Omega+\varepsilon \int_{\Omega}\left(\frac{|\nabla u|^{2}}{2}+\frac{|\nabla v|^{2}}{2}\right) d \Omega+\frac{K}{\varepsilon} \int_{\Omega} \frac{1}{2}\left(m \rho|\mathbf{v}|^{2}+\mathbf{S}: \mathbf{S}\right) d \Omega=0,
$$

что и означает единственность решения при малых значениях времени. Беря в качестве начального значения времени $t=1 / 2 L$ и продолжая этот процесс, получим единственность на все рассматриваемом промежутке времени $[0, \mathrm{~T}]$.

Замечание. Наличие конвективных слагаемых в уравнениях движения не препятствует получению нужных для единственности оценок. Однако присутствие в реологическом соотношении (уравнении состояния) производных от компонент тензора $\mathbf{S}$ по пространственным переменным не позволяет доказать единственность выбранным способом.

\section{Библиографический список}

1. Астарита Дж. Основы гидромеханики неньютоновских жидкостей / Дж. Астарита, Дж. Марручи. - М., 1978.

2. Joseph D. D. Fluid dynamics of viscoelastic fluids. N. Y., 1990.

3. Годунов С.К. Элементы механики сплошных сред и законы сохранения / С. К. Годунов, Е. И. Роменский. Новосибирск, 1998.

4. Звягин В.Г. Математические вопросы гидродинамики вязкоупругих сред / В.Г. Звягин, М.В. Турбин. - М., 2012.

5. Брутян М.А., Крапивский П.Л. Гидродинамика неньютоновских жидкостей // Комплексные и специальные разделы механики. - М., 1991. - Т. 4.
6. Gerritsma M.I., Phillips T.N. On the characteristics and compatibility equations for the UCM model fluid // Z. angew. Math. Mech. — 2008. — Bd 88, № 7.

7. Пухначев В.В. Математическая модель несжимаемой вязкоупругой среды Максвелла // ПМТФ. - 2010. - Т. 51, № 4.

8. Liapidevskii V.Yu., Pukhnachev V.V., Tani A. Nonlinear waves in incompressible viscoelastic Maxwell medium // Wave Motion. - 2011. - V. 48, iss. 8.

9. Мещерякова Е.Ю. Групповой анализ уравнений несжимаемой вязкоупругой среды Максвелла // Известия Алт. гос. ун-та. — 2012. — № 1/2.

10. Мелешко С.В., Петрова А.Г., Пухначев В.В. Характеристические свойства системы уравнений несжимаемой вязкоупругой среды Максвелла // ПМТФ. - 2017. - Т. 58, № 5. 